\title{
Prognosis in Pregnant Females With Systemic Lupus Erythematosus
}

\author{
Enling LIU, ${ }^{1}$ Yuxiu $\mathrm{ZHOU}^{2}$ \\ ${ }^{1}$ Department of Gynaecology and Obstetrics, Tangshan Clinical Medical College, Hebei Medical University, Tangshan, China \\ ${ }^{2}$ Department of Rheumatology, Tangshan Clinical Medical College, Hebei Medical University, Tangshan, China
}

\begin{abstract}
Objectives: This study aims to analyze the relationship between pregnancy and lupus, and explore the risk factors that adversely affect maternal and infant outcomes.

Patients and methods: The pregnancy outcomes in 112 pregnant females (mean age $24.3 \pm 2.8$ years; range 20 to 35 years) with systemic lupus erythematosus (SLE) were retrospectively analyzed. Pregnancy outcomes before and after pregnancy were compared, and the associations with lupus nephritis, positive anti-Ro/SSA antibody, positive La/SSB antibody, complement 3 and complement 4, high blood pressure, positive anticardiolipin ( $\mathrm{aCL}$ ) antibody, Raynaud's phenomenon, and lupus recurrence were evaluated. Factors contributing to adverse outcomes were analyzed using multinomial logistic regression.

Results: The live birth rate in females diagnosed with SLE before a pregnancy was higher than that in females diagnosed with SLE after a pregnancy. The fetal mortality rate in females diagnosed with SLE after a pregnancy was higher than that in females diagnosed with SLE before a pregnancy. However, the abortion rate in females diagnosed with SLE before a pregnancy was also significantly higher than that in females diagnosed with SLE after a pregnancy. The incidence of preterm birth in females diagnosed with SLE after a pregnancy was higher than that in females diagnosed with SLE after a pregnancy. Preterm birth was more likely to occur in females positive for Ro/SSA antibody. Patients with hypertension and Raynaud's phenomenon had a higher risk of intrauterine growth retardation. In addition, the presence of aCL antibody was associated with pregnancy loss. Multinomial logistic regression analysis showed that many factors might be associated with adverse pregnancy outcomes, including lupus nephritis, positive Ro/SSA antibody, positive La/SSB antibody, complement 3 and complement 4, positive aCL antibody, lupus recurrence, hypertension, and Raynaud's phenomenon.

Conclusion: Lupus nephritis, Ro/SSA antibody, aCL antibody, hypertension, Raynaud's phenomenon, and lupus recurrence are important factors associated with adverse pregnancy outcomes.

Keywords: Pregnancy outcome; pregnancy; prognostic factors; systemic lupus erythematosus.
\end{abstract}

Systemic lupus erythematosus (SLE) is a chronic autoimmune disease that is common in reproductive age groups, ${ }^{1}$ and may involve multiple organs, causing systemic damage. Pregnancy may induce lupus recurrence or exacerbation, leading to both maternal and infant injury. ${ }^{2-4}$ Such pregnancies are considered high-risk. The incidence rate of lupus recurrence is 13 to $68 \%$ during gestation, but is very low in the stationary phase. Pregnancy complicated by lupus can progress to miscarriage, premature birth, stillbirth, premature rupture of membranes, eclampsia, intrauterine growth retardation (IUGR), and neonatal lupus, and the rate of embryo loss is high in early pregnancy. Pregnancy can exacerbate lupus, and can even be life-threatening to mother and child. ${ }^{5-11}$ In this study, we aimed to analyze the relationship between pregnancy and lupus, and explore the risk factors that adversely affect maternal and infant outcomes.

\section{PATIENTS AND METHODS}

We retrospectively analyzed 112 pregnant females (mean age $24.3 \pm 2.8$ years; range 20 to 35 years) 
with SLE who gave birth in Tangshan Worker Hospital between January 2009 and February 2015. Of these, 100 patients were diagnosed with lupus before pregnancy (89.29\%), and 12 were diagnosed after they became pregnant. SLE was diagnosed according to the 1992 criteria of the American College of Rheumatology, based on clinical manifestations and the results of blood and urine analysis, including tests for antinuclear antibody, anti-double-stranded deoxyribonucleic acid antibody, anti-Sjögren's syndrome type A (anti-Ro/SSA) antibody, anti-Sjögren's syndrome type B (anti-La/SSB) antibody, anti-cardiolipin (aCL) antibody, and complement 3 (C3) and complement 4 (C4). All patients were managed by rheumatologists and obstetricians, according to immune and obstetric indices. Based on these indices, therapeutic doses of prednisone ranged from 5 to $20 \mathrm{mg} /$ day. The study protocol was approved by the Tangshan Worker Hospital Ethics Committee. A written informed consent was obtained from each patient. The study was conducted in accordance with the principles of the Declaration of Helsinki.

Pregnancy outcomes in females diagnosed with SLE before and after pregnancy were compared, and the associations with lupus nephritis, positive anti-Ro/SSA antibody, positive La/SSB antibody, $\mathrm{C} 3$ and $\mathrm{C} 4$, high blood pressure, positive $\mathrm{aCL}$ antibody, Raynaud's phenomenon, and lupus recurrence were evaluated. Generally, lupus recurrence was diagnosed when proteinuria increased, leukocytes and platelets reduced, and C3 decreased. Whether patients were treated with immunosuppressive agents such as cyclophosphamide before pregnancy was also considered. All immune indices, including Ro/SSA and $\mathrm{La} / \mathrm{SSB}$ antibodies, were measured using enzyme-linked immunosorbent assays, and C3 and $\mathrm{C} 4$ were measured with rate nephelometry.

Patients without recurrent symptoms were discharged to close observation and followup; patients who received prednisone and maintenance hydroxychloroquine sulfate before pregnancy remained on small doses of prednisone (5-10 mg/day) and hydroxychloroquine after delivery. SLE status was the basis for prednisone dose adjustment; the treatment protocol was adjusted according to the pre-pregnancy dose, and methylprednisolone was supplemented if necessary.

\section{Statistical analysis}

All data were analyzed using SPSS version 13.0 (SPSS Inc., Chicago, IL, USA) statistical software. Comparisons of continuous variables between two groups were performed using a t-test. Factors that adversely influenced pregnancy outcomes, including lupus nephritis, anti-Ro/SSA antibody, anti-La/SSB antibody, C3 and C4, anti$\mathrm{aCL}$ antibody, recurrence of lupus, hypertension, and Raynaud's phenomenon, were analyzed using multinomial logistic regression. $\mathrm{P}<0.05$ was considered statistically significant.

\section{RESULTS}

The 112 patients with SLE had a total of 131 pregnancies. The mean SLE duration was $10.2 \pm 3.6$ years (range: 2 to 17 years), with an average follow-up time of $6.3 \pm 4.1$ years (range: 0.5 to 16.5 years). Twelve cases of lupus (10.71\%) developed during pregnancy (lupus was diagnosed after pregnancy), and the other 100 cases (89.29\%) developed before pregnancy. Among 112 females with SLE, 91 were anti-double stranded deoxyribonucleic acid antibody-positive (81.25\%), 60 were anti-Ro/SSA antibody-positive (53.57\%), and 35 were anti-La/SSB antibodypositive (31.25\%); all 112 were antinuclear antibody-positive. There were 38 cases of a low level of C3 (33.93\%) and 32 of a low level of C4 (28.57\%). Among the 100 patients with SLE before pregnancy, 34 had lupus nephritis, 17 had high blood pressure, and 46 received cyclophosphamide before pregnancy.

Adverse pregnancy outcomes included abortion, stillbirth, neonatal death, miscarriage, preeclampsia, and IUGR. Among the 100 patients with SLE, 87 successfully gave birth to 98 babies, at a mean gestational age of $36.6 \pm 2.9$ weeks (range: 24 to 39 weeks). The average birth weight was $2.4 \pm 0.3 \mathrm{~kg}$ (range: 0.71 to $3.2 \mathrm{~kg}$ ). There were 15 cases of abortion, five of one miscarriage, and three of two or more miscarriages. There was one case of stillbirth and one of neonatal death. Of the 98 babies, 32 (32\%) had IUGR. Twelve patients with a total of 18 pregnancies had SLE recurrence after pregnancy. These females gave birth to 12 live infants and had six abortions, including four cases of one miscarriage and two of two or more miscarriages. Four newborns had 


\begin{tabular}{|lccc}
\hline Table 1. Influence factors of adverse pregnancy outcomes \\
\hline & OR & $95 \% \mathrm{CI}$ & $p$ \\
\hline Lupus nephritis & 7.2 & $1.2-41.8$ & 0.019 \\
Positive anti-aCL antibody & 3.8 & $1.1-13.3$ & 0.032 \\
Lupus recurrence & 1.9 & $1.2-3.2$ & 0.004 \\
Positive anti-La antibody & 11.4 & $1.1-115.1$ & 0.036 \\
Hypertension & 37.6 & $3.5-189.8$ & 0.002 \\
Raynaud's phenomenon & 12.4 & $2.3-69.9$ & 0.005 \\
\hline OR: Odds ratio; CI: Confidence interval; aCL: Anti-cardiolipin antibody. \\
\hline
\end{tabular}

growth delay, with an average gestational age of $37.5 \pm 3.2$ weeks (range: 27 to 38 weeks). The average birth weight was $3.1 \pm 0.8 \mathrm{~kg}$ (range: 0.84 to $3.17 \mathrm{~kg}$ ).

The live birth rate of females diagnosed with SLE after pregnancy was higher than that of females diagnosed with SLE before pregnancy $(p=0.048)$, as was the abortion rate $(50 \%$ vs. $15 \%$, respectively; $p=0.00062$ ). However, the fetal mortality rate and the incidence of preterm birth in patients diagnosed with SLE after pregnancy were higher than those of patients diagnosed with SLE before pregnancy $(p=0.026)$. Two pregnant females $(1.79 \%)$ died of lupus encephalopathy and multiple organ failure.

The live birth rate and rate of pregnancy loss in patients with lupus nephritis showed no significant difference between the two groups. However, patients who were anti-Ro/SSA antibody-positive were more likely to have preterm births $(p=0.014)$. In addition, patients with SLE with hypertension and Raynaud's phenomenon were at significantly higher risk for IUGR $(p=0.031)$, but those who were anti-La/SSB antibody-positive and had a low complement level did not have an adverse pregnancy outcome. The presence of anti-aCL antibody was more likely to lead to pregnancy loss $(\mathrm{p}=0.041)$.

We used multinomial logistic regression to explore factors leading to adverse pregnancy outcomes, including miscarriage, premature birth, and IUGR. Factors such as lupus nephritis, antiRo/SSA antibody, anti-La/SSB antibody, C3 and $\mathrm{C} 4$, anti-aCL antibody, lupus recurrence, hypertension, and Raynaud's phenomenon were analyzed. Among these, lupus nephritis (odds ratio $[\mathrm{OR}]=7.2 ; 95 \%$ confidence interval $[\mathrm{CI}]$ : 1.2-41.8; $\mathrm{P}=0.019)$, the presence of anti-aCL antibody (OR=3.8; 95\% CI: 1.1-13.3; $\mathrm{p}=0.032)$, and lupus recurrence $(\mathrm{OR}=1.9 ; 95 \% \mathrm{CI}$ : 1.2-3.2; $\mathrm{p}=0.004$ ) were important factors associated with fetal loss (abortion, stillbirth, and neonatal death); the presence of anti-La antibody $(\mathrm{OR}=11.4 ; 95 \%$ CI: 1.1-115.1; $\mathrm{p}=0.036)$, hypertension ( $\mathrm{OR}=37.6$; 95\% CI: 3.5-189.8; p=0.002), and Raynaud's phenomenon $(\mathrm{OR}=12.4$; 95\% CI: 2.3-69.9; $\mathrm{p}=0.005$ ) were factors with a potential association with IUGR (Table 1).

\section{DISCUSSION}

Systemic lupus erythematosus is an autoimmune disease with genetic heterogeneity. Patients with SLE develop a variety of autoantibodies that can form immune complexes and cause damage to multiple organs. Increased female hormones during gestation may lead to recurrence or exacerbation of lupus, and autoantibodies may be deposited in the kidney and heart. If thrombus forms in the placenta, abortion and stillbirth may result. It is believed that pregnancy does not change the long-term prognosis of SLE, but because SLE involves multiple organs, especially the kidney and heart, pregnancy may aggravate the burden on these organs, and eventually lead to recurrence or worsening of SLE. However, other researchers believe that antiphospholipid antibody and renal involvement in SLE do not affect pregnancy outcomes. ${ }^{12}$ With greater understanding of the interaction between pregnancy and SLE, as well as cooperation among physicians who provide care for such patients, the maternal and infant prognosis has greatly improved. ${ }^{13,14}$ However, some patients still have a poor prognosis, or even die of worsening SLE.

It is believed that SLE does not generally affect fertility in females, but the high doses of corticosteroids and immunosuppressants used 
in the active phase of SLE can cause menstrual disorders and affect ovarian function, which may reduce the fertility rate. SLE has an adverse effect on the progress and outcome of pregnancy. Thus, patients with SLE may have a high-risk pregnancy with an increased risk of complications, such as preeclampsia, premature rupture of membranes, fetal growth restriction, anemia, thrombocytopenia, postpartum hemorrhage, and delayed incision healing. ${ }^{15,16}$ Our results showed higher live birth rates after control of SLE, while pregnancy during an active SLE phase led to a higher incidence of pregnancy loss and preterm birth than that in patients with SLE diagnosed after pregnancy. Consistent with prior reports, newly-diagnosed SLE during pregnancy was more likely to involve the kidney and platelets. ${ }^{17-19}$ It was previously reported that the abortion rate in pregnant patients with SLE was 8 to $24 \%,{ }^{20}$ which was comparable to the $24.5 \%$ in this study. The incidence of premature births was $26.7 \%$. Some authors believe that lupus nephritis can result in an adverse maternal and infant outcome, ${ }^{21}$ but our results suggested that although lupus nephritis led to a higher incidence of preterm birth, the maternal and infant prognosis was good; this suggested that there was no clear correlation between abortion and lupus nephritis. The incidence of miscarriage in patients who were positive for anti-aCL antibody was two-fold higher than that in patients who were negative for anti-aCL antibody. Multinomial logistic regression analysis showed that the presence of anti-aCL antibody was strongly correlated with miscarriage, that anti-Ro/SSA antibody and high blood pressure were associated with prematurity, and that patients with high blood pressure and Raynaud's phenomenon had a high incidence of IUGR. However, the presence of anti-La/SSB antibody did not increase the risk of IUGR. Some studies showed that prednisone is effective in the treatment of SLE in pregnancy, and that pregnancy outcome is better than in patients who are not treated, but prednisone may lead to prematurity.

All 112 patients in this study were treated with oral prednisone and hydroxychloroquine. No fetal prematurity was found to be associated with the treatment.

Our results suggested that pregnancy during an active SLE phase was more likely to lead to pregnancy loss (including miscarriage, stillbirth, and neonatal death), premature birth, IUGR, and low birth weight. SLE can adversely affect the pregnancy and cause severe complications, such as lupus encephalopathy, pulmonary hypertension, multiple organ dysfunction, and increased maternal and infant mortality. The presence of lupus nephritis, anti-Ro/SSA antibody, anti-La/SSB, anti-aCL antibody, hypertension, Raynaud's phenomenon, and aggravated lupus were all predictors of poor pregnancy outcomes. Therefore, patients with SLE in reproductive age groups should receive active control of the disease. Pregnancy should only be considered after the disease is under control. Moreover, such patients should be closely monitored by an obstetrician and rheumatologist, and any abnormality should be promptly treated. If necessary, the pregnancy should be terminated to avoid serious maternal complications.

In conclusion, some factors that might contribute to adverse pregnancy outcomes include lupus nephritis, Ro/SSA antibody, aCL antibody, hypertension, Raynaud's phenomenon, and lupus recurrence.

\section{Declaration of conflicting interests}

The authors declared no conflicts of interest with respect to the authorship and/or publication of this article.

\section{Funding}

The authors received no financial support for the research and/or authorship of this article.

\section{REFERENCES}

1. Yang $\mathrm{H}$, Liu H, Xu D, Zhao L, Wang Q, Leng X, et al. Pregnancy-related systemic lupus erythematosus: clinical features, outcome and risk factors of disease flares--a case control study. PLoS One 2014;9:104375.

2. Stojan G, Baer AN. Flares of systemic lupus erythematosus during pregnancy and the puerperium: prevention, diagnosis and management. Expert Rev Clin Immunol 2012;8:439-53.

3. Perricone $\mathrm{C}$, de Carolis $\mathrm{C}$, Perricone R. Pregnancy and autoimmunity: a common problem. Best Pract Res Clin Rheumatol 2012;26:47-60.

4. Khamashta MA, Ruiz-Irastorza G, Hughes GR. Systemic lupus erythematosus flares during pregnancy. Rheum Dis Clin North Am 1997;23:15-30. 
5. Liu J, Zhao Y, Song Y, Zhang W, Bian X, Yang $\mathrm{J}$, et al. Pregnancy in women with systemic lupus erythematosus: a retrospective study of 111 pregnancies in Chinese women. J Matern Fetal Neonatal Med 2012;25:261-6.

6. Shand AW, Algert CS, March L, Roberts CL. Second pregnancy outcomes for women with systemic lupus erythematosus. Ann Rheum Dis 2013;72:547-51.

7. Tarr T, Kiss E, Szegedi G, Zeher M. Successful completed pregnancies in patients with systemic lupus erythematosus. Orv Hetil 2012;153:454-60. [Abstract]

8. Yan Yuen S, Krizova A, Ouimet JM, Pope JE. Pregnancy outcome in systemic lupus erythematosus (SLE) is improving: Results from a case control study and literature review. Open Rheumatol $\mathrm{J}$ 2008;2:89-98.

9. Ruiz-Irastorza G, Khamashta MA. Evaluation of systemic lupus erythematosus activity during pregnancy. Lupus 2004;13:679-82.

10. Kwok LW, Tam LS, Zhu T, Leung YY, Li E. Predictors of maternal and fetal outcomes in pregnancies of patients with systemic lupus erythematosus. Lupus 2011;20:829-36.

11. Märker-Hermann E, Fischer-Betz R. Rheumatic diseases and pregnancy. Curr Opin Obstet Gynecol 2010;22:458-65.

12. Madazli R, Bulut B, Erenel H, Gezer A, Guralp O. Systemic lupus erythematosus and pregnancy. J Obstet Gynaecol 2010;30:17-20.

13. Shen B, Tan W, Feng G, He Y, Liu J, Chen W, et al. The correlations of disease activity, socioeconomic status, quality of life, and depression/anxiety in Chinese patients with systemic lupus erythematosus. Clin Dev Immunol 2013;2013:270878.

14. Calixto OJ, Anaya JM. Socioeconomic status. The relationship with health and autoimmune diseases. Autoimmun Rev 2014;13:641-54.

15. Clowse ME. Lupus activity in pregnancy. Rheum Dis Clin North Am 2007;33:237-52.

16. Chakravarty EF, Colón I, Langen ES, Nix DA, El-Sayed YY, Genovese MC, et al. Factors that predict prematurity and preeclampsia in pregnancies that are complicated by systemic lupus erythematosus. Am J Obstet Gynecol 2005;192:1897-904.

17. Petri M. Prospective study of systemic lupus erythematosus pregnancies. Lupus 2004;13:688-9.

18. Molokhia M, Maconochie N, Patrick AL, Doyle P. Cross-sectional analysis of adverse outcomes in 1,029 pregnancies of Afro-Caribbean women in Trinidad with and without systemic lupus erythematosus. Arthritis Res Ther 2007;9:124.

19. Zhao C, Zhao J, Huang Y, Wang Z, Wang H, Zhang $\mathrm{H}$, et al. New-onset systemic lupus erythematosus during pregnancy. Clin Rheumatol 2013;32:815-22.

20. Teh CL, Wong JS, Ngeh NK, Loh WL. Systemic lupus erythematosus pregnancies: a case series from a tertiary, East Malaysian hospital. Lupus 2009;18:278-82.

21. Diniz-da-Costa T, Centeno M, Pinto L, Marques A, Mendes-Graça L. Systemic lupus erythematosus and pregnancy. Acta Med Port 2012;25:448-53. [Abstract] 